Relations industrielles

Industrial Relations

\title{
Mishel, Lawrence and Paula B. Voos, eds. Unions and Economic Competitiveness
}

\section{Claude Rondeau}

Volume 47, numéro 4, 1992

URI : https://id.erudit.org/iderudit/050819ar

DOI : https://doi.org/10.7202/050819ar

Aller au sommaire du numéro

Éditeur(s)

Département des relations industrielles de l'Université Laval

ISSN

0034-379X (imprimé)

1703-8138 (numérique)

Découvrir la revue

Citer ce compte rendu

Rondeau, C. (1992). Compte rendu de [Mishel, Lawrence and Paula B. Voos, eds. Unions and Economic Competitiveness]. Relations industrielles / Industrial

Relations, 47(4), 798-802. https://doi.org/10.7202/050819ar

Tous droits réservés (C) Département des relations industrielles de l'Université Laval, 1992
Ce document est protégé par la loi sur le droit d'auteur. L'utilisation des services d'Érudit (y compris la reproduction) est assujettie à sa politique d'utilisation que vous pouvez consulter en ligne.

https://apropos.erudit.org/fr/usagers/politique-dutilisation/ 
dominance of industrial relations in which unions are assigned an increasingly residual role. Another of these paths involves exist from the industry through, for example, bankruptcy or reestablishment in a new country. Yet another, along with auto manufacturing is allegedly headed, leads to joint labour-capital strategic initiatives at a higher level than conventional collective bargaining. These strategic alliances are the author's preferred route of evolution; one which they would like to see researched more thoroughly and supported by public policy. However, no overhaul of industrial relations is expected unless financial crises, as in steel, textiles, and airlines during the early 1990's, spur the search for alternatives to costly adversarialism. When no crisis occurs, as in education and telecommunications, the adversarial system continues to function, since both parties refuse to address innovations in their relationship which might alter the distribution of power to their disadvantage.

Chaykowski and Verma's refinement of the strategic-choice model is well grounded in the sector studies. The introduction of intermediate stages provides the strategic-choice model with a more dynamic character. Nevertheless, further elaboration of the intermediate stages may be necessary. For instance, human resource management (mining) and 'traditional' (construction and garments) forms of management dominance need to be distinguished, given their differing implications for industrial relations. In addition, the willingness of the actors to change their relationships (potential strategic alliances in telecommunications) in advance of expected crises (deregulation) should be recognized. Finally, the ability of the actors to prevent crises from occuring (non nonunion competition in education) should not be underestimated. However, these minor points do not detract from what is otherwise a valuable contribution to the literature and an accessible volume to all industrial relations specialists.

J. Mark HARCOURT

Edmonton, Alberta

Unions and Economic Competitiveness, Edited by de Lawrence Mishel and Paula B. Voos, Armonk, NY, M.E. Sharpe Inc., 1992, ISBN 0-87332-827-2 et ISBN 0-87332-828-0 (pbk)

La publication de Trade Unions in the Production Process par R. Freeman et J. Medoff en 1976 (traduit en français dans la Revue économique en 1979), a relancé le débat vigoureux qui oppose depuis toujours la vision institutionnaliste (plutôt positive) à la vision des économistes néo-classiques (plutôt négative), quant au rôle et à la contribution de la présence syndicale au fonctionnement du marché du travail et du régime de relations industrielles dans une économie industrialisée.

La contribution de Freeman et Medoff, cristallisée dans What do Unions Do? (1984), [Pourquoi les syndicats? (1985)], a été de fournir de nouvelles assises théoriques aux institutionnalistes, et d'en soumettre les prédictions à l'aune de l'analyse quantitative rigoureuse. Les articles rassemblés par Mishel et Voos dans ce volume collectif sont à la fois un prolongement et un élargissement de cet important et fructueux courant de pensée. Le champ de l'enquête est élargi de deux manières. La performance économique ne se limite plus à une 
mesure du niveau ou de la croissance de la productivité ou des profits, mais englobe la compétitivité sur les marchés mondiaux ainsi que l'ouverture au changement technologique. Au plan des institutions du marché du travail, des données aussi importantes que l'organisation du travail et les systèmes de rémunération s'ajoutent et s'associent à la présence syndicale pour contribuer à la performance économique, lorsque les conditions s'y prêtent. La notion et la nature de ces conditions favorables, désignées «climat des relations du travail» par Freeman et Medoff, demeurent toutefois une des principales faiblesses du Voice, qui n'est pas entièrement dissipée, et sur laquelle nous reviendrons. L'ouvrage permet aussi de progresser sur d'autres questions où Freeman et Medoff étaient vulnérables à la critique, comme celle de l'impact de longue période de la présence syndicale sur la croissance de la productivité et sur les investissements dans les entreprises syndiquées moins profitables que les autres.

Les auteurs sont clairement engagés à la recherche de retombées économiques positives de la présence syndicale dans l'économie américaine, aussi bien au plan macro-économique qu'au niveau de l'entreprise. Dans l'introduction, Mishel et Voos annoncent ainsi leurs couleurs et celles de leurs co-auteurs: développer et analyser l'hypothèse selon laquelle la présence syndicale peut améliorer plutôt que réduire la compétitivité de l'économie et des entreprises américaines. L'approche n'en est pas moins solide et rigoureuse, autant au plan théorique qu'à celui de la vérification empirique des hypothèses avancées, ce qui donne à l'ensemble un niveau de crédibilité très élevé.

Le volume comprend un substantiel chapitre d'introduction, suivi de huit textes répartis également en deux parties. La première partie est intitulée «les syndicats et l'économie». Les auteurs y reprennent les questions soulevées par Freeman et Medoff (1984), en élargissant la notion de performance économique. La deuxième partie, intitulée "Le rôle positif des syndicats", fait le point sur les questions nouvelles soulevées depuis quelques années dans l'évaluation de l'impact économique de la présence syndicale, comme l'organisation du travail, les systèmes de rémunération, et les relations coopératives entre employeur et syndicat au niveau de l'entreprise.

Examinant la notion de "compétitivité» des entreprises américaines sur les marchés mondiaux, l'introduction propose une distinction utile entre la compétitivité mesurée par les différences de coûts («cost competitiveness») et la compétitivité totale. Cette dernière tient compte, outre des différences de coûts, de différences dans d'autres facteurs comme le taux de change, la qualité du produit, et le design du produit. Au moyen de données comparatives sur les coûts relatifs du travail dans le secteur manufacturier et sur la structure des échanges tenant compte de la présence syndicale, Mishel et Voos montrent que la perte de compétitivité des États-Unis (i.e. le déficit de la balance commerciale) relativement à leurs concurrents, de 1973 à 1989, résulte de facteurs non associés directement aux coûts du travail. Ainsi mesurée, la perte de compétitivité s'observe autant dans les secteurs non syndiqués que dans les secteurs syndiqués.

C'est ainsi qu'ils argumentent que la présence syndicale, non seulement n'est pas responsable de la perte de compétitivité des entreprises américaines 
sur les marchés mondiaux, mais qu'elle pourrait contribuer à améliorer la situation.

En fait, selon eux, les Américains font face à un choix entre deux stratégies de production et de commercialisation: l'une, qui implique l'absence ou l'insignifiance des syndicats, est celle de la production de masse, à des salaires relativement bas; l'autre, qui repose sur l'appui de syndicats forts, dynamiques et respectés, est une stratégie de production axée sur la technologie de pointe à valeur ajoutée élevée. L'apport des syndicats à cette deuxième stratégie réside dans leur capacité à assurer les conditions nécessaires à l'essentielle présence d'une main-d'oeuvre flexible et hautement qualifiée, soit l'équité dans le partage des bénéfices, la sécurité d'emploi et le Voice dans les milieux de travail.

Karier présente une analyse plus approfondie de l'impact de la présence syndicale dans les entreprises américaines sur le déficit de la balance commerciale, pour l'ensemble de l'économie et par branches d'industrie. II examine aussi l'hypothèse de la fuite à l'étranger des entreprises américaines pour éviter la présence syndicale et les différences de coûts qui y sont associées. Dans les trois cas, il conclut des données examinées que ce n'est pas la présence syndicale, mais d'autres facteurs, qui peuvent être à l'origine des déficits commerciaux.

Le quatrième article de la première partie est une mise à jour de What Do Unions Do? par Freeman. Selon ce dernier, neuf des principales conclusions de cet ouvrage sont confirmées huit ans plus tard. Plusieurs autres n'ont pas été remises en question, particulièrement celles sur le roulement de la maind'oeuvre. Quatre conclusions nouvelles sont tirées des recherches plus récentes: une association positive entre la présence syndicale, et l'innovation en matière de technologie et de relations du travail, la flexibilité des salaires en récession, ainsi que le taux d'épargne chez les cols bleus; une association négative avec les dépenses des entreprises en recherches et développement. Nul doute que l'idée d'une plus grande flexibilité des salaires chez les syndiqués que les non syndiqués en fera sursauter certains; selon Freeman, cette constatation reflète les concessions salariales acceptées par les syndicats au cours de la dernière décennie. Or, ces concessions ont résulté tout autant de pressions structurelles que conjoncturelles, et elles ne font pas nécessairement la preuve d'une flexibilité conjoncturelle des salaires des travailleurs syndiqués.

Par ailleurs, selon Freeman la robustesse des conclusions de What Do Unions Do? soutient l'idée, non seulement d'une contribution positive de la présence syndicale à la performance économique, mais aussi de l'absence de substitut à cette présence. Ainsi, selon lui, il faudrait prendre des mesures aux États-Unis pour renverser le déclin du syndicalisme. II reconnaît néanmoins que personne n'a encore expliqué comment se développe au niveau de l'entreprise le climat de relations coopératives du travail dont dépend en définitive la contribution positive de la présence syndicale.

Les deux autres articles de la première partie sont parmi les plus importants de ce volume. Dans le premier, Belman présente une synthèse de la littérature sur l'impact de la présence syndicale sur la productivité (niveau et croissance), sur les profits, ainsi que sur l'étude du climat de relations du travail. 
Les cinq annexes ( 31 pages) qui résument sous forme de tableau schématique les 55 études recensées valent à elles seules l'achat du volume. Dans le second, Keefe aborde la question difficile et controversée de la contribution positive ou négative de la présence syndicale au changement technologique. II s'agit d'un volet crucial de la stratégie de production axée sur la technologie de pointe, nécessitant une main-d'oeuvre flexible et d'un haut niveau de qualification. D'une revue de la littérature théorique et empirique, Keef conclut que les entreprises utilisent davantage la technologie de pointe lorsqu'elles sont syndiquées (mais non à cause de la présence syndicale), et que les syndicats acceptent et parfois soutiennent activement le changement technologique lorsque la sécurité d'emploi et de revenu de leurs membres est raisonnablement assurée. Une des conclusions les plus intéressantes de Keefe est que l'encadrement juridique des relations du travail, et les politiques managériales de protection des droits de la direction, peuvent faire obstacle à une plus grande participation des syndicats aux décisions en matière de choix technologiques en les reléguant à un rôle passif, et peuvent rendre le changement technologique plus difficile pour cette raison.

Cette question de l'importance de la qualité des relations du travail pour le changement technologique est reprise par Kelley et Harrison dans un texte de la deuxième partie du volume. Ces auteurs examinent plus particulièrement l'apport positif de relations coopératives à cet égard. Ils présentent une synthèse théorique fort remarquable des liens entre la participation des employés et la performance d'entreprise. Ils soutiennent ensuite que la présence syndicale est un rouage essentiel à une contribution positive de la participation des employés à la performance de l'entreprise.

Un autre texte de la deuxième partie ressort plus particulièrement, celui de Eaton et Voos. Dans cet article, les auteures proposent une classification des innovations en matière d'organisation du travail, de rémunération et de participation (cette classification est en elle-même un apport utile; le lecteur appréciera aussi le glossaire et la revue de littérature en annexe). Elles cherchent ensuite à déterminer quelles innovations contribuent le plus à améliorer la productivité ou la performance de l'entreprise. Elles proposent une conclusion qui sera d'une grande importance si elle est confirmée par d'autres études: les innovations en relations du travail sont plus nombreuses et plus profondes en milieu syndiqué, et sont plus susceptibles d'accroître la productivité, parce qu'elles s'appuient sur des relations institutionnalisées entre la direction de l'entreprise et le syndicat, ce qui favorise la coopération et un engagement correspondant des employés. En milieu non syndiqué, les innovations les plus répandues sont la participation aux profits ou l'actionnariat ouvrier, des mesures passives qui s'adressent aux employés à titre individuel, et qui n'ont que peu d'impact sur la productivité.

Le troisième texte de la deuxième partie propose aux Américains un examen de l'expérience allemande, en alternative au Japon, comme source d'inspiration en matière d'innovations en relations du travail. Selon Turner, la tradition institutionnelle des États-Unis, particulièrement en ce qui a trait au syndicalisme, se rapproche davantage de celle de l'Europe occidentale que de celle du Japon. II explique comment les syndicats allemands ont été intégrés et même assimilés, sans être défaits, à l'appareil de gestion économique, aussi bien au plan macro-économique qu'au niveau de l'entreprise. Cette opération, 
basée en partie sur la prise en charge des organes de la co-détermination par les syndicats, a permis à l'économie allemande, et particulièrement à l'industrie de l'automobile, de se restructurer de façon cohérente avec la pleine participation des syndicats. Comparativement, Turner décrit la dispersion des mesures d'adaptation prises dans l'industrie américaine de l'automobile. Selon lui, cette dispersion reflète les contradictions dans les politiques des employeurs, aussi bien à l'égard de la présence syndicale que des innovations en matière de relations du travail, ainsi que l'instabilité de la représentation syndicale qui en résulte. Bref, par l'étude du cas allemand, Turner cherche à montrer que l'amélioration de la performance économique ne nécessite pas de casser les syndicats (approche américaine), ni de chercher un syndicalisme subordonné et soumis (approche japonaise). Au contraire, la présence d'un syndicalisme fort et indépendant est non seulement compatible avec des mesures de réorganisation du travail qui accroissent la productivité, mais peut même améliorer l'efficacité de telles mesures.

Enfin, le texte de Marshall, un ancien ministre du travail, prend les allures d'un programme politique qui s'appuie toutefois sur une analyse sérieuse et étoffée des options qui s'offrent en matière de relations du travail aux ÉtatsUnis. Invoquant les changements structurels et technologiques survenus depuis dix ou quinze ans, il reprend l'idée d'un choix stratégique, avancée par les éditeurs du volume dans l'introduction, entre une économie axée sur la production de masse et les bas salaires, ou orientée vers la technologie de pointe, et des niveaux élevés de qualité, de productivité et de qualifications du travail. La deuxième stratégie est préférable. Toutefois, elle ne peut réussir sans la participation des employés, laquelle nécessite une représentation institutionnelle indépendante et puissante.

Cet ouvrage marque une étape importante dans la recherche de plus en plus abondante sur ce qu'on peut maintenant appeler l'économie de la représentation syndicale. II systématise et synthétise les connaissances sur plusieurs aspects, notamment l'impact de la présence syndicale sur la productivité. Surtout, il apporte un éclairage nouveau sur les interactions entre la présence syndicale dans l'entreprise et la compétitivité sur les marchés mondiaux, le changement technologique et l'efficacité d'innovations en relations du travail ou en gestion des ressources humaines comme la participation, la rémunération flexible, le travail en équipe, etc...

En justifiant théoriquement l'apport positif de la présence syndicale, certains des auteurs, particulièrement Keefe sur le changement technologique, ainsi que Kelley et Harrison et Eaton et Voos sur les innovations en relations du travail, ont réussi à entrouvrir la fameuse boîte noire dans laquelle se génèrent les bonnes relations du travail nécessaires à la performance économique au niveau de l'entreprise. II reste néanmoins du travail à faire pour expliquer pourquoi certains couples employeur-syndicat ont des relations coopératives ou harmonieuses, tandis que d'autres, pourtant semblables en apparence, s'en tiennent à la confrontation constante et hargneuse.

Claude Rondeau 